\title{
An Electrothermal Carbon Nanotube Gas Sensor
}

\author{
Takeshi Kawano, Heather C. Chiamori, Marcel Suter, Qin Zhou, \\ Brian D. Sosnowchik, and Liwei Lin* \\ Berkeley Sensor and Actuator Center, Department of Mechanical Engineering, \\ University of California at Berkeley, California 94720
}

Received August 7, 2007; Revised Manuscript Received October 15, 2007

\begin{abstract}
We show both gas pressure and species sensing capabilities based on the electrothermal effect of a multiwalled carbon nanotube (MWCNT). Upon exposure to gaseous environments, the resistance of a heated MWCNT is found to change following the conductive heat-transfer variances of gas molecules. To realize this mechanism, a suspended MWCNT is constructed by synthesis and assembly in localized chemical vapor deposition that is accomplished within seconds via real-time electrical feedback control. Vacuum pressure sensitivity and gas species differentiability are observed and analyzed. Such MWCNT electrothermal sensors are compact, fast and reversible in responses, and fully integratable with microelectronics.
\end{abstract}

Carbon nanotube (CNT) gas sensors based on fundamentally different mechanisms have been demonstrated, ${ }^{1}$ including electrical conductance or capacitance changes ${ }^{2-4}$ or using the sharp tips of the CNT as gas ionization sensors. ${ }^{5}$ Limiting factors for sorption-based CNT sensors include low adsorption energies, diffusion kinetics, response speed, selectivity, and reversibility. We report on the electrothermal effect of a single multiwalled carbon nanotube (MWCNT) suspended between two silicon microbridges. When a Joule-heated MWCNT is exposed to different pressures, the electrical resistance change follows the conductive heat-transfer variances of gas molecules. The MWCNT is grown on silicon using the local synthesis method and is accomplished within tens of seconds via real-time electrical feedback control. Our findings suggest that the MWCNT electrothermal mechanism has applications to both gas sensing and species differentiation, with advantages of compactness, fast and reversible responses, low power consumption, and being fully integratable with microelectronics.

The electrothermal gas sensing mechanism is schematically illustrated in Figure 1a. A single MWCNT is suspended between two silicon microstructures and heated by electrical current in the system. Energy conservation calls for total heat generation equal to the summation of heat conduction to the two microstructures $\left(W_{\mathrm{C}}\right)$, heat transfer via gases $\left(W_{\mathrm{G}}\right.$, shown in the figure as $W_{\text {gas1 }}$ and $W_{\text {gas2 }}$ representing the case of two types of gases of different thermal conductivity values), and heat radiation $\left(W_{\mathrm{R}}\right)$. Both gas pressure and species can affect

* To whom correspondence should be addressed. Telephone: 1-(510) 643-5495. Fax: 1-(510) 643-5599. E-mail: 1wlin@me.berkeley.edu. the heat-transfer process and result in MWCNT temperature changes and therefore resistance changes.

To realize this architecture, we use a complementary metal oxide semiconductor (CMOS)-compatible, in situ controlled synthesis, assembly, and integration process previously reported in the literature. ${ }^{6-9}$ Synthesis and assembly of carbon nanotubes has been heavily investigated with various device demonstrations using high-temperature synthesis processes ${ }^{10,11}$ and labor-intensive assembly steps, ${ }^{12,13}$ but assembly and heterogeneous integration of CNTs with microelectronics such as standard CMOS circuitry are still problematic. Many issues still need to be resolved, such as the synthesis and accurate placement of CNTs at specific locations, controllable and in situ verification of assembly with good electrical/ mechanical contacts, and fast and direct synthesis with widely available, prefabricated microelectronics. ${ }^{14}$ As a further improvement, we have added an in situ monitoring capability used in conjunction with the localized synthesis process. The inset image of the voltage versus time plot (Figure 1b) shows that when a CNT connection is made across the silicon microstructures, there is a corresponding voltage jump. By the number of voltage increases, we can determine the number of successful CNT connections. Figure 1c shows two successfully synthesized and assembled examples. Sample A was synthesized and assembled in approximately $8 \mathrm{~s}$. Sample B has the first and second MWCNTs synthesized and assembled within 20 and $50 \mathrm{~s}$, respectively. Figure 1d shows a scanning electron microscope (SEM) image of a single, $25 \mu \mathrm{m}$ long, suspended MWCNT that is synthesized and self-assembled between two silicon microstructures. We have accomplished several CNT samples that exhibit both 
(a)

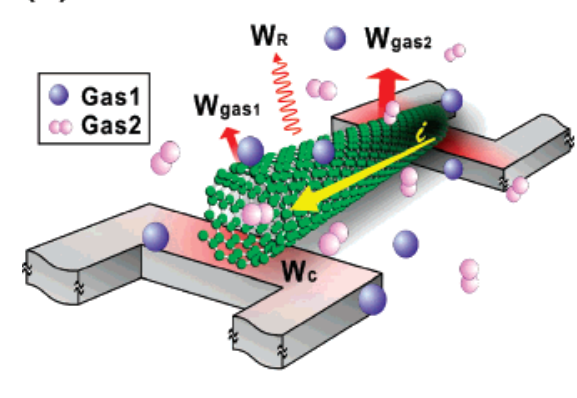

(c)

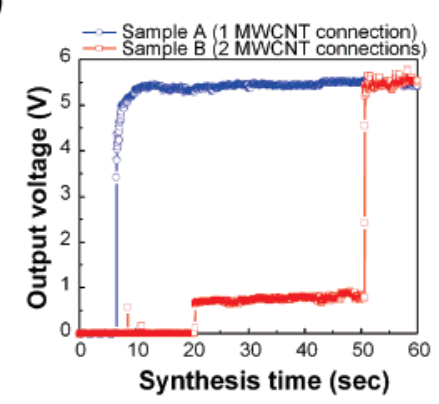

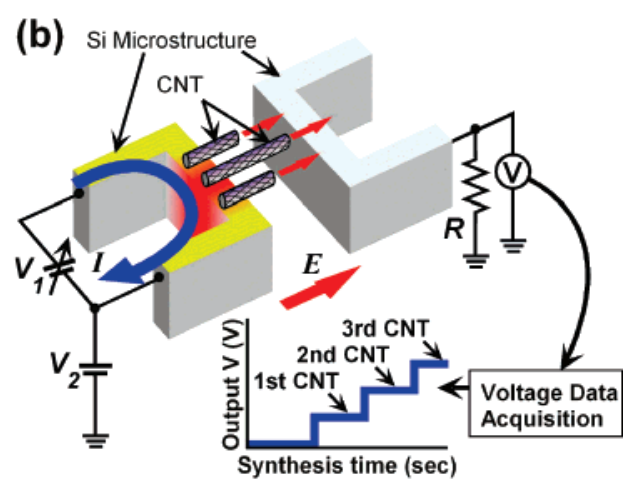

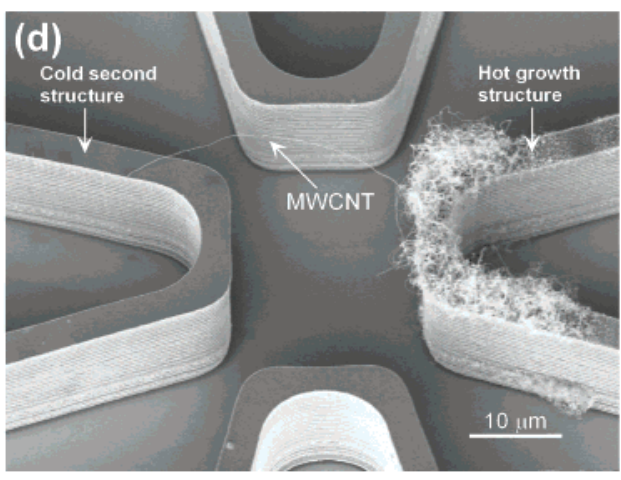

Figure 1. CNT electrothermal gas sensing and local synthesis and assembly of a single MWCNT. (a) Schematic diagram showing the architecture and gas sensing principle of the suspended CNT. Heat generation in a CNT by the applied current is dissipated by heat conduction to gases ( $\left.W_{\text {gas } 1}, W_{\text {gas } 2}\right)$, heat radiation $\left(W_{\mathrm{R}}\right)$, and heat conduction to the contact $\left(W_{\mathrm{C}}\right)$ with the supporting microstructure. (b) The schematic setup of local synthesis and assembly by local electrical-field-guided chemical vapor deposition. The hot growth structure activates the MWCNT growth, and the cold second structure provides bias for the local electrical field and real-time electrical feedback. (c) Two successful samples showing connection of one (circles) and two (squares) MWCNTs (here, bias $V_{1}=7.5 \mathrm{~V}$ and $V_{2}=5 \mathrm{~V}$ ). (d) SEM image of a single MWCNT electrothermal gas sensor.

gas pressure and species sensing capabilities. All experimental data presented here are from the MWCNT sample shown in Figure 1d.

Current-voltage $(I-V)$ characteristics of this suspended MWCNT sample are measured immediately after the synthesis process in an argon environment under pressures of $10^{5}$ and $6.5 \times 10^{3} \mathrm{~Pa}$ (Figure 2a). The linear behavior in both cases under low input power implies ohmic contact, while the nonlinear behavior at higher power densities can be analyzed with an analytical model similar to that of a thermal conductivity gauge or Pirani gauge. ${ }^{15,16}$ To characterize the temperature resistance responses, the resistances of this single MWCNT have been measured over the range of 200 to $400 \mathrm{~K}$, and the extracted temperature coefficient of resistance (TCR) has shown linear behavior as $-0.137 \% \mathrm{~K}^{-1}$ (See Figure 5 in Supporting Information). On the basis of this information, a $1 \mathrm{~V}$ bias voltage applied to the MWCNT in an argon environment is estimated to increase the average MWCNT temperatures by 40 and $140 \mathrm{~K}$ over the ambient temperature under the pressures of $10^{5} \mathrm{~Pa}$ and $6.5 \times 10^{3} \mathrm{~Pa}$, respectively. Although a previous report shows that a suspended metallic single-walled CNT (SWCNT) has a negative differential conductance due to electron scattering by hot nonequilibrium optical phonons under a much higher power density, ${ }^{17}$ this phenomenon has not been explored here as our sensor works under low power density for moderate operating temperatures.

The MWCNT resistance changes are further characterized as a function of input voltage in argon under various pressures (Figure 2b). In the molecular regime, gas density is low, and the molecules are assumed to experience essentially no collisions among themselves as they travel along the path between the MWCNT and the surrounding walls. Gas molecules arriving at the hot wire will have a Maxwellian energy distribution corresponding to $T_{0}$ (ambient temperature), as explained in the classic thermal conductivity gauge theories, ${ }^{15,16}$ and these molecules dwell on the surface for a short time and depart with an energy distribution corresponding to $T_{\mathrm{CNT}}$. The heat transfer via gas between a MWCNT and an imaginary coaxial cylindrical wall can be approximate as ${ }^{18}$

$$
W_{\mathrm{G}}=\frac{1}{4} \frac{(\gamma+1)}{(\gamma-1)} \alpha \sqrt{\frac{2 R}{\pi M T_{0}}}\left(T_{\mathrm{CNT}}-T_{0}\right) p
$$

where $p$ is the pressure, $\gamma$ the ratio of specific heats of the gas, $R$ the universal gas constant, and $M$ the molecular mass of the gas. The thermal accommodation coefficient, $\alpha$, describes the heat transfer from the MWCNT to the gas molecule and depends on the type of gas. It is observed that $W_{\mathrm{G}}$ is proportional to pressure $p$ in this regime if all other parameters are constants. Under higher input voltage, $T_{\mathrm{CNT}}$ is expected to be higher, causing $W_{\mathrm{G}}$ to be higher with larger resistance drops for better pressure sensitivity $(\Delta R / \Delta p)$, as observed.

A single-stage amplifier is used to obtain the voltage signals across the MWCNT in a SEM (LEO 1550). The Pirani gauge of the SEM sends signals ranging from 
(a)

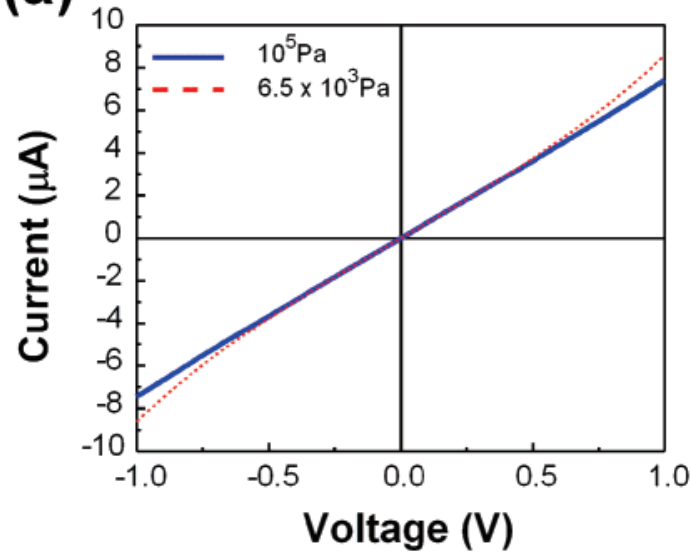

(b)

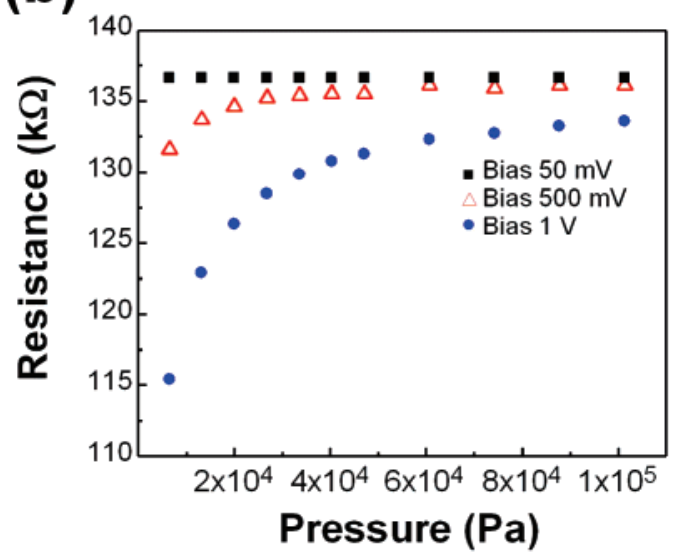

Figure 2. Electrothermal characteristics of the suspended MWCNT. (a) Current versus voltage curves recorded in $10^{5}$ (solid line) and $6.5 \times 10^{3}$ (dash line) $\mathrm{Pa}$ argon environments, showing the resistance changes due to gas thermal conductivity. (b) Resistance versus pressure curves recorded under $1,0.5$, and $0.05 \mathrm{~V}$ of input voltages in an argon environment, indicating higher sensitivity under higher bias voltage.

approximately $10^{4}$ to $10^{-1} \mathrm{~Pa}$; then, the ion gauge initiates signals for pressures under $10^{-1} \mathrm{~Pa}$. The MWCNT was active from $10^{2}$ to $10^{5} \mathrm{~Pa}$ and consistently converged under high vacuum conditions (Figure 3a). Five sets of data were recorded repeatedly using alternating nitrogen and air environments, with consistent results and no observable oxygen absorption effects. As shown in Figure $3 b$, the voltage-pressure plot can reveal information about the energy-transfer processes. At low pressures, $\left(W_{\mathrm{C}}+W_{\mathrm{R}}\right)$ determines the baseline energy loss of the MWCNT and the lowest measurable pressure level of the gas sensor. ${ }^{19}$ From our experimental data and assuming an idealized model, values for $W_{\mathrm{C}}, W_{\mathrm{R}}$, and $W_{\text {TOTAL }}$ can be determined at the baseline pressures. The energy loss due to radiation is approximated using eq 2

$$
W_{\mathrm{R}}=\epsilon \sigma\left(T_{\mathrm{CNT}}^{4}-T_{0}^{4}\right) A
$$

where $\sigma$ is the Stefan-Boltzmann constant, $A$ is the crosssectional area of the MWCNT, and $\epsilon$ is the emissivity, here, considered unity. $W_{\text {TOTAL }}$ can be represented by the total
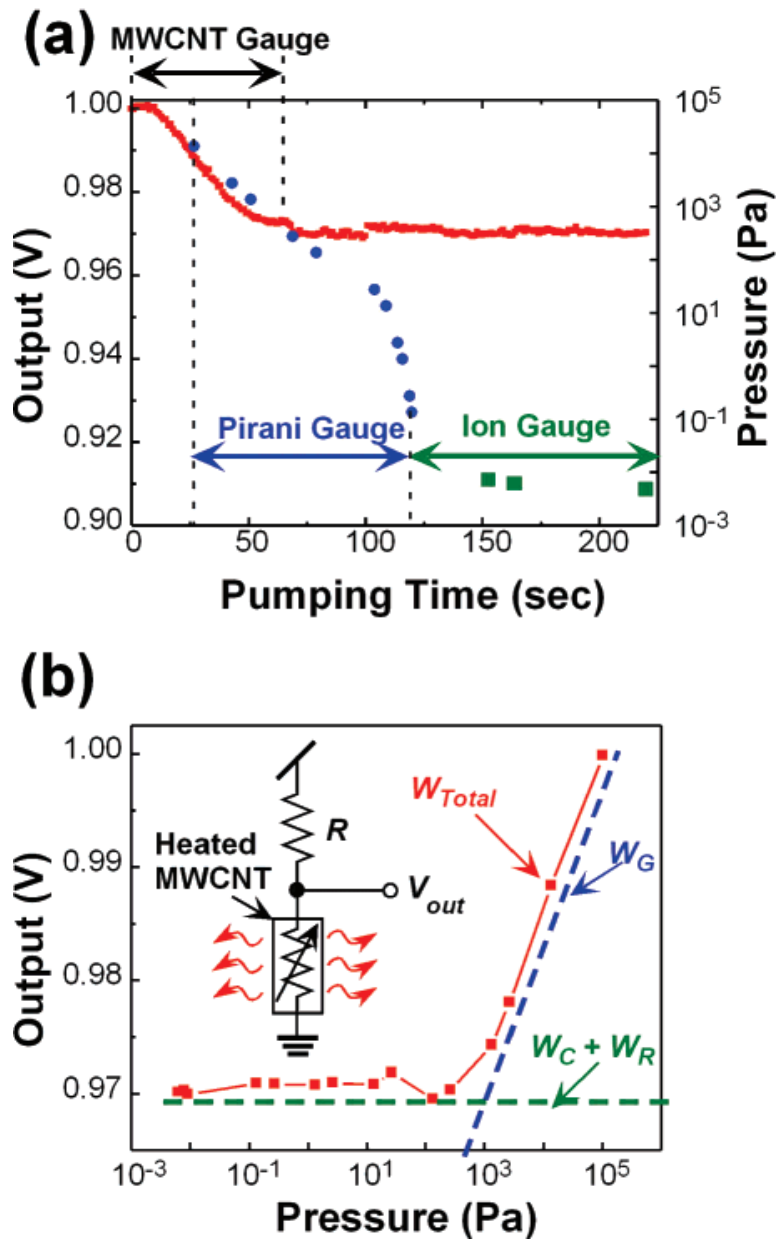

Figure 3. Voltage outputs from a single-stage amplifier for the MWCNT in SEM (LEO 1550) during the dual-stage pump-down process. (a) Voltage output versus time plot showing pressure readouts of the MWCNT (red), SEM Pirani gauge (circles), and SEM ion gauge (squares). The MWCNT provides readings between $10^{2}$ to $10^{5} \mathrm{~Pa}$ consistently. (b) Voltage versus pressure curve indicating the pressure detection and limit of the MWCNT. The lower pressure limit is determined by the heat conduction to the microstructure via the contacts $\left(W_{\mathrm{C}}\right)$ and radiation $\left(W_{\mathrm{R}}\right)$, suggesting that a long-wire, small MWCNT contact area and low thermal conductivity of the MWCNT could be preferred to have more sensitive gas pressure sensing capability.

power input to the system and is obtained experimentally. The energy loss through the contacts is then estimated using $W_{\mathrm{C}}=W_{\mathrm{TOTAL}}-W_{\mathrm{R}}$. We estimate that the radiation energy loss is less than $20 \mathrm{nW}$, which is considered negligible such that the energy loss through the contacts is approximately $W_{\text {TOTAL}}$, or $7.5 \mu \mathrm{W}$. A simplified heat conduction equation with a first-order approximation based on a symmetrical temperature profile on the MWCNT can be shown as

$$
\frac{1}{2} W_{\mathrm{C}}=\left.k_{\mathrm{CNT}} A \frac{\mathrm{d} T_{\mathrm{CNT}}}{\mathrm{d} l}\right|_{l=0}
$$

where $k_{\mathrm{CNT}}$ is the thermal conductivity of the MWCNT, $A$ is the interfacial contact area between the MWCNT and the silicon microstructure, and $\mathrm{d} T_{\mathrm{CNT}} /\left.\mathrm{d} l\right|_{l=0}$ is the slope of the temperature profile beginning at the MWCNT/silicon interface on the growth structure. The MWCNT temperature 
(a)

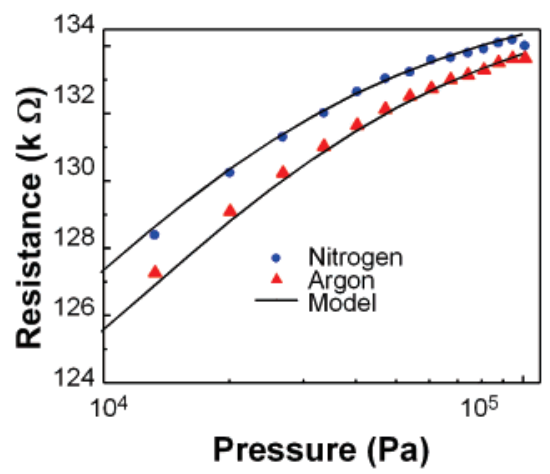

(b)

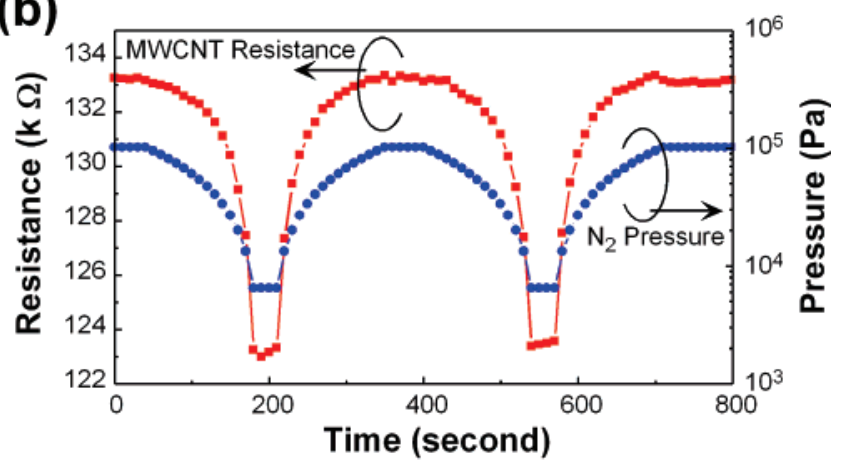

Figure 4. Differentiation of gas species and sensing reversibility and repeatability. (a) Resistance change versus pressure in nitrogen and argon environments as compared with the analytical model. Different gas responses with varying pressure are consistent with the analytical model that is constructed by using the known gas thermal conductivity values. (b) Continuous resistance change versus time with a pressure control of every $10 \mathrm{~s}$ in a nitrogen environment. No delay is observed, indicating a fast response time, which cannot be explained by previously reported gas sensing mechanisms.

profile can be simulated using an energy balance between the energy provided by resistive heating of the system and the total energy losses represented by $W_{\mathrm{C}}, W_{\mathrm{R}}$, and $W_{\mathrm{G}}$. A value for $k_{\mathrm{CNT}}$ can be extracted using eq 3 and the estimated value for $W_{\mathrm{C}}$ at the lower pressure sensing limit of the MWCNT. Our simplified model yields a thermal conductivity value for $k_{\mathrm{CNT}}$ of approximately $300 \mathrm{~W} / \mathrm{mK} .{ }^{20}$ Previous theoretical and experimental reports on $k_{\mathrm{CNT}}$ show values as high as $6600 \mathrm{~W} / \mathrm{mK}^{21}$ and as low as $25 \mathrm{~W} / \mathrm{mK}^{22}$ with other values in-between. ${ }^{23,24}$ Here, defects from the growth process may contribute to the low thermal conductivity value. Additionally, this lower value of $k_{\mathrm{CNT}}$ is advantageous for the electrothermal pressure sensing process. Theoretically, smaller values of $W_{C}$ will extend the lower pressure limit of the MWCNT, which requires a lower $k_{\mathrm{CNT}}$, smaller contact area, and less steep temperature gradient at the contacts. A longer MWCNT could be preferable to provide a less steep temperature gradient under the same operating conditions.

The heated MWCNT sample also shows the capability of differentiating argon and nitrogen gases at various pressures (Figure 4a). Previously, a metallic SWCNT has demonstrated gas species differentiation under high power density in $1 \mathrm{~atm}$ of pressure by the principle of relaxing hot optical phonons, while the gas thermal conductivity was not a primary factor. ${ }^{25}$ Large $W_{\mathrm{C}}$ values from the short SWCNT length used in this previous report could be the reason that gas thermal conductivity values were not a factor. Here, we find that the thermal conductivity value in nitrogen $(25.83 \mathrm{~mW} / \mathrm{m} \cdot \mathrm{K})$ and $\operatorname{argon}(17.72 \mathrm{~mW} / \mathrm{m} \cdot \mathrm{K})$ is the key to differentiate these two gas species, similar to that of a conventional thermal conductivity gauge. ${ }^{19,26}$ Simulation results show consistency using the characteristics of thermal conductivity (see Supporting Information and Figure 4a). In practice, conventional Pirani gauges have been used in gas chromatographs to differentiate gas species. The electrothermal CNT sensor could offer several advantages in compactness, low power consumption, and fast response time..$^{27}$ The electrothermal sensing mechanism does not rely on gas adsorption; thus, the response speed can be fast, with good reversibility without memory effects, as demonstrated in a continuous operation in nitrogen pressure cycles for $800 \mathrm{~s}$ (Figure 4b). Data were collected every $10 \mathrm{~s}$ as limited by our current capability of changing the pressure levels. These initial promising results indicate that further reducing the contact area, increasing the CNT length, and a lower thermal conductivity value, combined with improved external circuit design such as constant-temperature mode and Wheatstone bridge configuration, can result in a new class of thermal conductivity-based gas sensing systems based on the electrothermal resistive effect of the CNT.

Acknowledgment. We thank Dr. C. Yu for the measurement of the MWCNT temperature coefficient of resistance. We are grateful for the contributions and helpful discussions of D. Christensen and C.Y. Cho at the University of California at Berkeley. T. Kawano is funded by the JSPS Postdoctoral Fellowships for Research Abroad from Japan. H. Chiamori is a recipient of the NSF Fellowship. This work is supported, in part, under DARPA MTO MEMS/NEMS S\&T program and a NSF Grant EEC-0425914.

Supporting Information Available: Sample preparation, TCR characterization, and the electrothermal model. This material is available free of charge via the Internet at http:// pubs.acs.org.

\section{References}

(1) Baughman, R. H.; Zakhidov, A. A.; de Heer, W. A. Science 2002, 297, 787.

(2) Collins, P. G.; Bradley, K.; Ishigami, M.; Zettl, A. Science 2000, 287, 1801.

(3) Kong, J.; Franklin, N. R.; Chongwu, Z.; Chapline, M. G.; Peng, S.; Cho, K.; Dai, H. Science 2000, 287, 622.

(4) Snow, E. S.; Perkins, F. K.; Houser, E. J.; Badescu, S. C.; Reineckeet, T. L. Science 2005, 307, 1942.

(5) Modi, A.; Koratkar, N.; Lass, E.; Wei, B.; Ajayan, P. M. Nature 2003, 424, 171.

(6) Englander, O.; Christensen, D.; Lin, L. Appl. Phys. Lett. 2003, 82, 4797.

(7) Englander, O.; Christensen, D.; Kim, J.; Lin, L.; Morris, S. J. S. Nano Lett. 2005, 5, 705.

(8) Kawano, T.; Christensen, D.; Chen, S.; Cho, C. Y.; Lin, L. Appl. Phys. Lett. 2006, 89, 163510.

(9) Englander, O.; Christensen, D.; Kim, J.; Lin, L. Sens. Actuators, A 2007, 135, 10.

(10) Iijima, S. Nature 1991, 354, 56.

(11) Ebbesen, T. W. Carbon Nanotubes: Preparation and Properties; CRC Press, Boca Raton, FL, 1997.

(12) Tans, S. J.; Devoret, M. H.; Dai, H.; Thess, A.; Smalley, R. E.; Geerligs, L. J.; Dekker, C. Nature 1997, 386, 474. 
(13) Bachtold, A.; Hadley, P.; Nakanishi, T.; Dekker, C. Science 2001, 294, 1317.

(14) Tseng, Y. C.; Xuan, P.; Javey, A.; Malloy, R.; Wang, Q.; Bokor, J.; Dai, H. Nano Lett. 2004, 4, 123.

(15) Lafferty, J. Foundations of Vacuum Science and Technology; John Wiley \& Sons, Inc.: New York, 1998.

(16) Bermann, A. Total Pressure Measurements in Vacuum Technology; Academic Press Inc.: New York, 1985.

(17) Pop, E.; Mann, D.; Cao, J.; Wang, Q.; Goodson, K.; Dai, H. Phys. Rev. Lett. 2005, 95, 155505.

(18) Knudsen, M. Ann. Phys. (Leipzig) 1911, 34, 593.

(19) Ellefson, R. E.; Miiller, A. P. J. Vac. Sci. Technol. 2000, A18, 2568.

(20) On the basis of the assumption that the MWCNT has an outer diameter of $30 \mathrm{~nm}$ and an inner diameter of $10 \mathrm{~nm},\left\{\mathrm{~d} T_{\mathrm{CNT}}\right\} /\left.\{\mathrm{d} l\}\right|_{l=0}$ is estimated at $2 \times 10^{7} \mathrm{~K} / \mathrm{m}$.

(21) Berber, S.; Kwon, Y.; Tomanek, D. Phys. Rev. Lett. 2000, 84, 4613.

(22) Yi, W.; Lu, L.; Dian-lin, Z.; Pan, Z. W.; Xie, S. S. Phys. Rev. B 1999, 59, R9015.

(23) Hone, J.; Whitney, M.; Piskoti, C.; Zettl, A. Phys. Rev. B 1999, 59 , $\mathrm{R} 2514$.

(24) Kim, P.; Shi, L.; Majumdar, A.; McEuen, P. L. Phys. Rev. Lett. 2001 $87,215502$.
(25) Mann, D.; Pop, E.; Cao, J.; Wang, Q.; Goodson, K.; Dai, H. J. Phys. Chem. B 2006, 110, 1502

(26) Leck, J. H. Total and Partial Pressure Measurement in Vacuum Systems; Kluwer: Norwell, MA, 1989.

(27) For a first-order transient analysis, we consider the MWCNT thermal capacity as an electrical capacitor and MWCNT thermal resistance to the gas as a series electrical resistor, with $L$ as a unit length scale. Changes in pressure will cause heat-transfer changes in a manner similar to charge transfer in the resistor-capacitor circuit such that the time constant is proportional to the multiple of resistance and capacitance. Since thermal resistance is inversely proportional to area or $L^{-2}$ and the heat capacitor is proportional to the volume or $L^{3}$, the resulting time response is proportional to $L$. In the example of a traditional Pirani gauge with a diameter of tens of micrometers compared with a MWCNT diameter of tens of nanometers, we would expect a three-order faster thermal time response for the MWCNT.

NL071964S 